\title{
Some more results on fuzzy $k$-competition graphs
}

\author{
Sovan Samanta \\ Department of Applied \\ Mathematics with Oceanology \\ and Computer Programming, \\ Vidyasagar University, \\ Midnapore - 721 102, India. \\ email: ssamantavu@gmail.com
}

\author{
Madhumangal Pal \\ Department of Applied \\ Mathematics with Oceanology \\ and Computer Programming, \\ Vidyasagar University, \\ Midnapore - 721 102, India. \\ email: mmpalvu@gmail.com
}

\author{
Anita Pal
}

\begin{abstract}
Fuzzy competition graph as the generalization of competition graph is introduced here. Fuzzy $k$-competition graph as a special type of fuzzy competition graph is defined here along with fuzzy isolated vertices. The fuzzy competition number is also introduced and investigated several properties. Isomorphism properties on fuzzy competition graphs are discussed.
\end{abstract}

Keywords: Fuzzy graphs, fuzzy competition graphs, fuzzy $k$-competition graphs, fuzzy competition number.

\section{INTRODUCTION}

The concept of fuzzy graph was introduced by Rosenfeld [36] in 1975. Fuzzy graph theory has a vast area of applications. It is used in evaluation of human cardiac function, fuzzy neural networks, etc. Fuzzy graphs can be used to solve traffic light problem, time table scheduling, etc. In fuzzy set theory, there are different types of fuzzy graphs which may be a graph with crisp vertex set and fuzzy edge set or fuzzy vertex set and crisp edge set or fuzzy vertex set and fuzzy edge set or crisp vertices and edges with fuzzy connectivity, etc. A lot of works have been done on fuzzy graphs [5], [6], [14], [20], [22], [26].

In 1968, Cohen [12] introduced the notion of competition graphs in connection with a problem in ecology. Let $\vec{D}=$ $(V, \vec{E})$ be a digraph, which corresponds to a food web. A vertex $x \in V(\vec{D})$ represents a species in the food web and an arc $\overrightarrow{(x, s)} \in \vec{E}(\vec{D})$ means that $x$ preys on the species $s$. If two species $x$ and $y$ have a common prey $s$, they will compete for the prey $s$. Based on this analogy, Cohen defined a graph which represents the relations of competition among the species in the food web. The competition graph $C(\vec{D})$ of a digraph $\vec{D}=(V, \vec{E})$ is an undirected graph $G=(V, E)$ which has the same vertex set $V$ and has an edge between two distinct vertices $x, y \in V$ if there exists a vertex $s \in$ $V$ and $\operatorname{arcs} \overrightarrow{(x, s)}, \overrightarrow{(y, s)} \in \vec{E}(\vec{D})$. The competition graph is also applicable in channel assignment, coding, modelling of complex economic and energy systems, etc. [35].

A lot of works have been done on competition graphs and its variations. In all these works, it is assumed that the vertices and edges of the graphs are precisely defined. But, in reality we observe that sometimes the vertices and edges of a graph can not be defined precisely. For example, in ecology, species may be of different types like vegetarian, non-vegetarian, strong, weak, etc. Similarly in ecology, preys may be tasty, digestive, harmful, etc. The terms tasty, digestive, harmful, etc. have no precise meanings. They are fuzzy in nature and hence the species and preys may be assumed as fuzzy sets and inter-relationship between the species and preys can be designed by a fuzzy graph. This motivates the necessity of fuzzy competition graphs.

The competition graphs and fuzzy graphs are well known topics. In this article, fuzzy competition graphs are defined as motivated from fuzzy food web. Also, the generalization of it, the fuzzy $k$-competition graphs is introduced. Fuzzy neighbourhood graphs and their properties are investigated. Fuzzy competition number and fuzzy isolated vertex are exemplified. Isomorphism of fuzzy competition graphs is discussed.

\section{A. Review of literature}

In 1968, Cohen [12] presented a nice technique for food webs to find minimum number of dimensions of niche spaces and defined competition graphs. After Cohen's introduction of competition graph, several variations of it are found in literature. These are $p$-competition graphs of a digraph [16], [19], tolerance competition graphs [9], $m$-step competition graphs of a digraph [11], competition hypergraphs [46], common enemy graph of a digraph [21], competition-common enemy graph of a digraph [45], etc. Surveys of the large literature related to competition graphs can be found in [10], [15], [43]. All these representations are crisp in sense and do not include all real field competitions. The competition graphs describe about the common prey and related species, but they do not measure the strength of competitions. These graphs do not show that how much the species depend on a common prey compared to other species. On the other hand fuzzy graph theory, after Rosenfeld [36], is increased with a large number of branches [2], [3], [4], [27], [28], [29], [30], [32], [34], [37], [38].

To include the representations of all real world competitions, fuzzy competition graphs are introduced. The relations among neighbourhoods of any species are described in fuzzy neighbourhood graphs. Sometimes the preys are so valuable that the species (competitors) compete strongly. Different level 
of competitions can shown by "fuzzy $k$-competition graphs", a generalization of fuzzy competition graph which is introduced here.

\section{PRELIMINARIES}

\section{A. Competition graphs}

A directed graph (digraph) $\vec{G}$ is a graph which consists of non-empty finite set $V(\vec{G})$ of elements called vertices and a finite set $\vec{E}(\vec{G})$ of ordered pairs of distinct vertices called arcs. We will often write $\vec{G}=(V, \vec{E})$. For an arc $\overrightarrow{(u, v)}, u$ is the tail and $v$ is the head. The order (size) of $\vec{G}$ is the number of vertices (arcs) in $\vec{G}$. The out-neighbourhood [17] of a vertex $v$ is the set $N^{+}(v)=\{u \in V-v: \overrightarrow{(v, u)} \in \vec{E}\}$. Similarly, the in-neighbourhood [17] $N^{-}(v)$ of a vertex $v$ is the set $\{w \in$ $V-v: \overrightarrow{(w, v)} \in \vec{E}\}$. The open neighbourhood of a vertex is the union of out-neighbourhood and in-neighbourhood of the vertex. A walk in $\vec{G}$ is an alternating sequence $W=$ $x_{1} \overrightarrow{e_{1}} x_{2} \overrightarrow{e_{2}} \ldots x_{k-1} \overrightarrow{e_{k}} x_{k}$ of vertices $x_{i}$ and arcs $\overrightarrow{e_{i}}$ of $\vec{G}$ such that tail of $\overrightarrow{e_{i}}$ is $x_{i}$ and head is $x_{i+1}$ for every $i=1,2, \ldots, k-$ 1. A walk is closed if $x_{1}=x_{k}$. A trail is a walk in which all arcs are distinct. A path is a walk in which all vertices are distinct. A path $x_{1}, x_{2}, \ldots, x_{k}$ with $k \geq 3$ is a cycle if $x_{1}=x_{k}$.

For an undirected graph, open-neighbourhood [1] $N(x)$ of the vertex $x$ is the set of all vertices adjacent to $x$ in the graph. Open neighbourhood graph [1] $N(G)$ of $G$ is a graph whose vertex set is same as $G$ and has an edge between two vertices $x$ and $y$ in $N(G)$ if and only if $N(x) \cap N(y) \neq \phi$ in $G$. Closed neighbourhood $N[x]$ of $x$ is the set $N(x) \cup\{x\}$. Closed neighbourhood graph $N[G]$ of a graph $G$ is similarly defined, except has an edge in $N[G]$ if and only if $N[x] \cap N[y] \neq \phi$ in $G$. $(p)$-neighbourhood graph (read as open $p$-neighbourhood graph) [8], $N_{p}(G)$ of a graph $G$ is a graph whose vertex set is same as $G$ and has an edge between two vertices $x$ and $y$ if and only if $|N(x) \cap N(y)| \geq p$ (note that $|X|$ is the number of elements in the crisp set $X$ ) in $G$. Similarly $[p]$ neighbourhood graph (closed $p$-neighbourhood graph) $N_{p}[G]$ [8] is defined similar in $N_{p}(G)$ except there is an edge between $x$ and $y$ if and only if $|N[x] \cap N[y]| \geq p$ in $G$.

Definition 1: [12] The competition graph $C(\vec{G})$ of a digraph $\vec{G}=(V, \vec{E})$ is an undirected graph $G=(V, E)$ which has the same vertex set $V$ and has an edge between distinct two vertices $x, y \in V$ if there exist a vertex $a \in V$ and $\operatorname{arcs} \overrightarrow{(x, a)}, \overrightarrow{(y, a)} \in \vec{E}$ in $\vec{G}$. We say that a graph $G$ is a competition graph if there exists a digraph $\vec{G}$ such that $C(\vec{G})=G$.

Many variations of competition graph have been available in literature [9], [11], [16], [19], [46]. One of the important graphs, known as $p$-competition graphs, is defined below .

Definition 2: [19] If $p$ is a positive integer, the $p$ competition graph $C_{p}(\vec{G})$ corresponding to the digraph $\vec{G}$ is defined to have a vertex set $V$ with an edge between $x$ and $y$ in $V$ if and only if, for some distinct vertices $a_{1}, a_{2}, \ldots, a_{p}$ in
$V, \overrightarrow{\left(x, a_{1}\right)}, \overrightarrow{\left(y, a_{1}\right)}, \overrightarrow{\left(x, a_{2}\right)}, \overrightarrow{\left(y, a_{2}\right)}, \ldots, \overrightarrow{\left(x, a_{p}\right)}, \overrightarrow{\left(y, a_{p}\right)}$ are arcs in $\vec{G}$.

If $\vec{G}$ is thought of as a food web whose vertices are the species in some ecosystem, $(x, y)$ is an edge of $C_{p}(\vec{G})$ if and only if $x$ and $y$ have at least $p$ common preys. So $C_{1}(\vec{G})$ is the competition graph.

\section{B. Fuzzy graphs}

A fuzzy set $A$ on a set $X$ is characterized by a mapping $m: X \rightarrow[0,1]$, which is called the membership function. A fuzzy set is denoted by $A=(X, m)$. The support of $A$ is supp $A=\{x \in X \mid m(x) \neq 0\}$. The core of $A$ is the crisp set of all members whose membership values are $1 . A$ is non trivial if supp $A$ is non-empty. The height of $A$ is $h(A)=\max \{m(x) \mid x \in X\} . A$ is normal if $h(A)=1$. The membership function of the intersection of two fuzzy sets $A$ and $B$ with membership functions $m_{A}$ and $m_{B}$ respectively is defined as the minimum of the two individual membership functions. $m_{A \cap B}=\min \left\{m_{A}, m_{B}\right\}$. We write $A=(X, m)$ $\leq B=\left(X, m^{\prime}\right)$ (fuzzy subset) if $m(x) \leq m^{\prime}(x)$ for all $x \in$ $X$. The family of all fuzzy subsets is denoted by $\mathcal{F}(x)$. The cardinality of a fuzzy set $A=(X, m)$ is a positive real number $c(A)$ or $|A|$ is the sum of membership values of the elements of $X$ [?]. Now the fuzzy graph is defined below.

Definition 3: [36] A fuzzy graph $\xi=(V, \sigma, \mu)$ is a nonempty set $V$ together with a pair of functions $\sigma: V \rightarrow[0,1]$ and $\mu: V \times V \rightarrow[0,1]$ such that for all $x, y \in V$, $\mu(x, y) \leq \sigma(x) \wedge \sigma(y)$ and $\mu$ is a symmetric fuzzy relation on $\sigma$. Here $\sigma(x)$ and $\mu(x, y)$ represent the membership values of the vertex $x$ and of the edge $(x, y)$ in $\xi$.

Since $\mu$ is well defined, a fuzzy graph has no multiple edges. A loop at a vertex $x$ in a fuzzy graph is represented by $\mu(x, x) \neq 0$. The fuzzy set $(V, \sigma)$ is called fuzzy vertex set of $\xi$ and the elements of the fuzzy set are called fuzzy vertices. $(V \times V, \mu)$ is called the fuzzy edge set of $\xi$ and the elements of the fuzzy set are called fuzzy edge. An edge is non-trivial if $\mu(x, y) \neq 0$. The fuzzy graph $\xi^{\prime}=\left(V^{\prime}, \tau, \nu\right)$ is called a fuzzy subgraph [24] of $\xi$ if $\tau(x) \leq \sigma(x)$ for all $x \in V^{\prime}$ and $\nu(x, y) \leq \mu(x, y)$ for all $x, y \in V^{\prime}$ where $V^{\prime} \subset V$.

For the fuzzy graph $\xi=(V, \sigma, \mu)$, an edge $(x, y), x, y \in V$ is called strong [13] if $\frac{1}{2} \min \{\sigma(x), \sigma(y)\} \leq \mu(x, y)$ and it is called weak otherwise.

Like crisp digraphs there is fuzzy digraphs which are defined below.

Definition 4: [25] Directed fuzzy graph (fuzzy digraph) $\vec{\xi}=(V, \sigma, \vec{\mu})$ is a non-empty set $V$ together with a pair of functions $\sigma: V \rightarrow[0,1]$ and $\vec{\mu}: V \times V \rightarrow[0,1]$ such that for all $x, y \in V, \vec{\mu}(x, y) \leq \sigma(x) \wedge \sigma(y)$.

Since $\vec{\mu}$ is well defined, a fuzzy digraph has at most two directed edges (which must have opposite directions) between any two vertices. Here $\vec{\mu}(u, v)$ is denoted by the membership value of the edge $\overrightarrow{(u, v)}$. The loop at a vertex $x$ is represented by $\vec{\mu}(x, x) \neq 0$. Here $\vec{\mu}$ need not be symmetric as $\vec{\mu}(x, y)$ and $\vec{\mu}(y, x)$ may have different values. The underlying crisp graph of directed fuzzy graph is the graph similarly obtained except the directed arcs are replaced by undirected edges. 


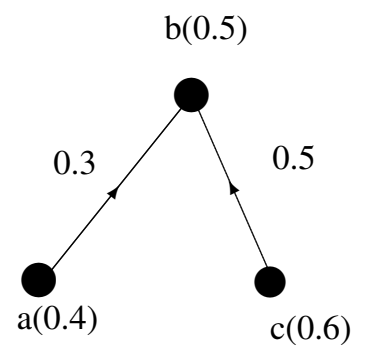

Fig. 1. Example of fuzzy out-neighbourhood and in-neighbourhood of a vertex

A homomorphism [27] between fuzzy graphs $\xi$ and $\xi^{\prime}$ is a map $h: S \rightarrow S^{\prime}$ which satisfies $\sigma(x) \leq \sigma^{\prime}(h(x))$ for all $x \in S$ and $\mu(x, y) \leq \mu^{\prime}(h(x), h(y))$ for all $x, y \in S$ where $S$ is set of vertices of $\xi$ and $S^{\prime}$ is that of $\xi^{\prime}$.

A weak isomorphism [27] between fuzzy graphs is a bijective homomorphism $h: S \rightarrow S^{\prime}$ which satisfies $\sigma(x)=$ $\sigma^{\prime}(h(x))$ for all $x \in S$.

A co-weak isomorphism [27] between fuzzy graphs is a bijective homomorphism $h: S \rightarrow S^{\prime}$ which satisfies $\mu(x, y)=$ $\mu^{\prime}(h(x), h(y))$ for all $x, y \in S$.

An isomorphism [27] between fuzzy graphs is a bijective homomorphism $h: S \rightarrow S^{\prime}$ which satisfies $\sigma(x)=\sigma^{\prime}(h(x))$ for all $x \in S$ and $\mu(x, y)=\mu^{\prime}(h(x), h(y))$ for all $x, y \in S$.

\section{FUZZY COMPETITION GRAPHS}

Now, we come to our main objective of the paper, the fuzzy competition graph. Like crisp graph, fuzzy out-neighbourhood and fuzzy in-neighbourhood of a vertex in directed fuzzy graph are defined below.

Definition 5: Fuzzy out-neighbourhood of a vertex $v$ of a directed fuzzy graph $\vec{\xi}=(V, \sigma, \vec{\mu})$ is the fuzzy set $\mathcal{N}^{+}(v)=\left(X_{v}^{+}, m_{v}^{+}\right)$where $X_{v}^{+}=\{u \mid \vec{\mu}(v, u)>0\}$ and $m_{v}^{+}: X_{v}^{+} \rightarrow[0,1]$ defined by $m_{v}^{+}(u)=\vec{\mu}(v, u)$. Similarly, fuzzy in-neighbourhood of a vertex $v$ of a directed fuzzy graph $\vec{\xi}=(V, \sigma, \vec{\mu})$ is the fuzzy set $\mathcal{N}^{-}(v)=\left(X_{v}^{-}, m_{v}^{-}\right)$where $X_{v}^{-}=\{u \mid \vec{\mu}(u, v)>0\}$ and $m_{v}^{-}: X_{v}^{-} \rightarrow[0,1]$ defined by $m_{v}^{-}(u)=\vec{\mu}(u, v)$.

Example 1: Let $\vec{\xi}$ be a directed fuzzy graph. Let the vertex set be $\{a, b, c\}$ with membership values $\sigma(a)=0.4$, $\sigma(b)=0.5, \sigma(c)=0.6$. The membership values of arcs are $\vec{\mu}(a, b)=0.3, \vec{\mu}(c, b)=0.5$. So $\mathcal{N}^{+}(a)=\{(b, 0.3)\}$. $\mathcal{N}^{-}(b)=\{(a, 0.3),(c, 0.5)\}$. (Note that $(a, \sigma(a))$ represents the vertex $a$ with membership value $\sigma(a)$ ). It is shown in Figure 1.

Now, we define fuzzy competition graph.

Definition 6: The fuzzy competition graph $\mathcal{C}(\vec{\xi})$ of a fuzzy digraph $\vec{\xi}=(V, \sigma, \vec{\mu})$ is an undirected fuzzy graph $\xi=$ $(V, \sigma, \mu)$ which has the same fuzzy vertex set as in $\vec{\xi}$ and has a fuzzy edge between two vertices $x, y \in V$ in $\mathcal{C}(\vec{\xi})$ if and only if $\mathcal{N}^{+}(x) \cap \mathcal{N}^{+}(y)$ is non-empty fuzzy set in $\vec{\xi}$. The edge membership value between $x$ and $y$ in $\mathcal{C}(\vec{\xi})$ is $\mu(x, y)=(\sigma(x) \wedge \sigma(y)) h\left(\mathcal{N}^{+}(x) \cap \mathcal{N}^{+}(y)\right)$.

Example 2: Let $\vec{\xi}$ be a directed fuzzy graph. Let the vertices with membership values of $\vec{\xi}$ be $(a, 0.3),(b, 0.6)$,

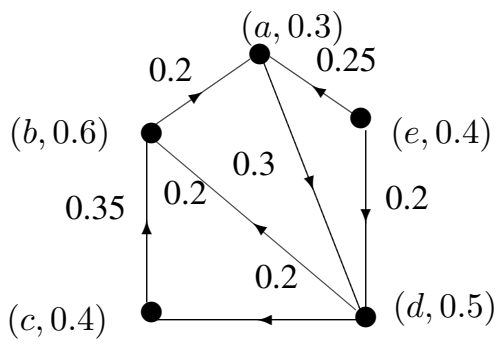

$\vec{\xi}$

(a) : Directed fuzzy graph

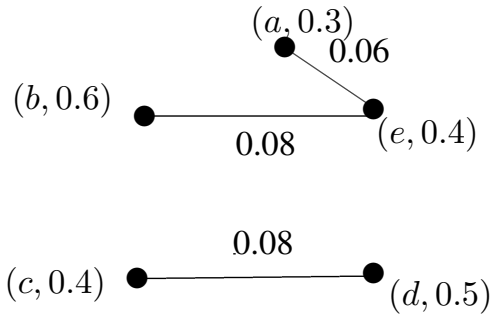

$C(\vec{\xi})$

(b) : Fuzzy competition graph

Fig. 2. Example of fuzzy competition graph

$(c, 0.4),(d, 0.5),(e, 0.4)$ with membership values of arcs be $\vec{\mu}(b, a)=0.2, \vec{\mu}(c, b)=0.35, \vec{\mu}(d, c)=0.2, \vec{\mu}(e, d)=$ $0.2, \vec{\mu}(e, a)=0.25, \vec{\mu}(a, d)=0.3$ (shown in Figure 2(a)). The corresponding fuzzy competition graph is shown in Figure 2(b).

Edge in fuzzy competition graphs indicates that there is a competition between two vertices (species) for at least one prey. So the strengths of the edges are important to characterize the competitions.

Now an extension of fuzzy competition graph, called fuzzy $k$-competition graph is defined in the following.

Definition 7: Let $k$ be a non-negative number. The fuzzy $k$ competition graph $\mathcal{C}_{k}(\vec{\xi})$ of a fuzzy digraph $\vec{\xi}=(V, \sigma, \vec{\mu})$ is an undirected fuzzy graph $\xi=(V, \sigma, \mu)$ which has the same fuzzy vertex set as $\vec{\xi}$ and has a fuzzy edge between two vertices $x, y \in V$ in $\mathcal{C}_{k}(\vec{\xi})$ if and only if $\left|\mathcal{N}^{+}(x) \cap \mathcal{N}^{+}(y)\right|>$ $k$. The edge membership value between $x$ and $y$ in $\mathcal{C}_{k}(\vec{\xi})$ is $\mu(x, y)=\frac{\left(k^{\prime}-k\right)}{k^{\prime}}[\sigma(x) \wedge \sigma(y)] h\left(\mathcal{N}^{+}(x) \cap \mathcal{N}^{+}(y)\right)$ where $k^{\prime}=\left|\mathcal{N}^{+}(x) \cap \mathcal{N}^{+}(y)\right|$.

So fuzzy $k$-competition graph is simply fuzzy competition graph when $k=0$. An example of fuzzy 0.15 -competition graph is given below.

Example 3: Let $\vec{\xi}$ be a directed fuzzy graph (Figure 3(a)). Let vertices with membership values of $\vec{\xi}$ be $(x, 0.4)$, $(y, 0.6),(a, 0.6),(b, 0.7),(c, 0.8)$, and the membership values of arcs be $\vec{\mu}(x, a)=0.3, \vec{\mu}(x, b)=0.35, \vec{\mu}(x, c)=0.36$, 


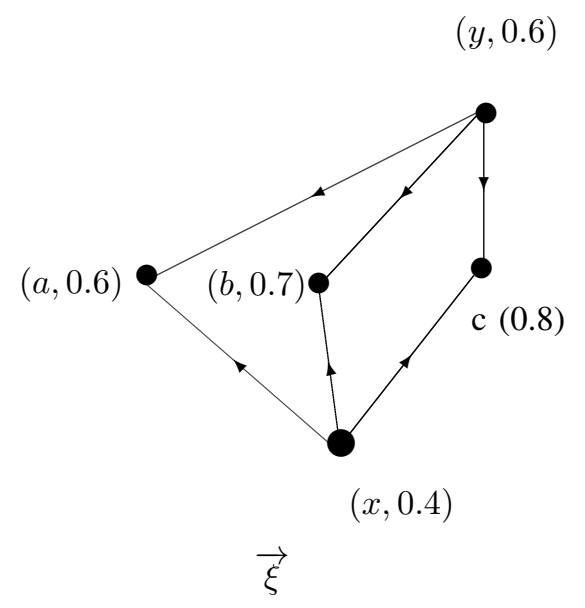

(a): Fuzzy directed graph

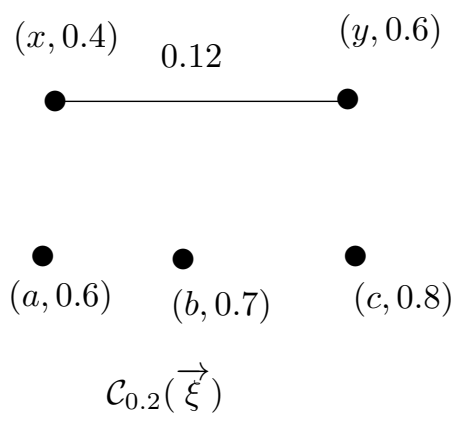

(b): Fuzzy 0.15-competition graph

Fig. 3. Example of fuzzy 0.15-competition graph

$\vec{\mu}(y, a)=0.6, \vec{\mu}(y, b)=0.5, \vec{\mu}(y, c)=0.45$. The corresponding fuzzy 0.15 -competition graph is shown in Figure 3(b).

Theorem 1: Let $\vec{\xi}=(V, \sigma, \vec{\mu})$ be a fuzzy digraph. If $\mathcal{N}^{+}(x) \cap \mathcal{N}^{+}(y)$ is singleton set, then the edge $(x, y)$ of $C(\vec{\xi})$ is strong if and only if $\left|\mathcal{N}^{+}(x) \cap \mathcal{N}^{+}(y)\right|>0.5$.

Proof. Here $\vec{\xi}=(V, \sigma, \vec{\mu})$ is a fuzzy digraph. Let $\mathcal{N}^{+}(x) \cap$ $\mathcal{N}^{+}(y)=\{(a, m)\}$, where $m$ is the membership value of the element $a$. Here, $\left|\mathcal{N}^{+}(x) \cap \mathcal{N}^{+}(y)\right|=m=h\left(\mathcal{N}^{+}(x) \cap\right.$ $\left.\mathcal{N}^{+}(y)\right)$. So, $\mu(x, y)=m \times \sigma(x) \wedge \sigma(y)$. Hence the edge $(x, y)$ in $C(\vec{\xi})$ is strong if and only if $m>0.5$.

If all the edges of a fuzzy digraph are strong, then all the edges of the corresponding fuzzy competition graph may not be strong. This result is illustrated below. Let us consider two vertices $x, y$ with $\sigma(x)=0.3, \sigma(y)=0.4$ in a fuzzy digraph such that the vertices have a common prey $z$ with $\sigma(z)=0.2$. Let $\vec{\mu}(x, z)=0.2, \vec{\mu}(y, z)=0.15$. Clearly, the edges $\overrightarrow{(x, z)}$ and $(y, z)$ are strong. But membership value of the edge $(x, y)$ in corresponding competition graph is $0.3 \times 0.15=0.045$. Hence the edge is not strong as $\frac{0.045}{0.3}=0.15<0.5$. But if all the edges are strong of a fuzzy digraph, then a result can be found from the following theorem.

Theorem 2: If all the edges of a fuzzy digraph $\vec{\xi}=$ $(V, \sigma, \vec{\mu})$ be strong, then $\frac{\mu(x, y)}{(\sigma(x) \wedge \sigma(y))^{2}}>0.5$ for all edge $(x, y)$ in $C(\vec{\xi})$.

Proof. Let $\vec{\xi}=(V, \sigma, \vec{\mu})$ be a fuzzy digraph and every edge of $\vec{\xi}$ be strong i.e., $\frac{\vec{\mu}(x, y)}{\sigma(x) \wedge \sigma(y)}>0.5$ for all edge $(x, y)$ in $\vec{\xi}$. Let the corresponding fuzzy competition graph be $C(\vec{\xi})=$ $(V, \sigma, \mu)$.

Case 1: Let $\mathcal{N}^{+}(x) \cap \mathcal{N}^{+}(y)$ be a null set for all $x, y \in V$. Then there exist no edge in $C(\vec{\xi})$ between $x$ and $y$.

Case 2: $\mathcal{N}^{+}(x) \cap \mathcal{N}^{+}(y)$ is not a null set. Let $\mathcal{N}^{+}(x) \cap$ $\mathcal{N}^{+}(y)=\left\{\left(a_{1}, m_{1}\right),\left(a_{2}, m_{2}\right), \ldots,\left(a_{z}, m_{z}\right)\right\}$, where $m_{i}, i=$ $1,2, \ldots, z$ are the membership values of $a_{i}, i=1,2, \ldots, z$, respectively. So $m_{i}=\min \left\{\vec{\mu}\left(x, a_{i}\right), \vec{\mu}\left(y, a_{i}\right)\right\}, i=$ $1,2, \ldots, z$. Let $h\left(\mathcal{N}^{+}(x) \cap \mathcal{N}^{+}(y)\right)=\max \left\{m_{i}, i=\right.$ $1,2, \ldots, z\}=m_{\max }$.

$\mu(x, y)=(\sigma(x) \wedge \sigma(y)) h\left(\mathcal{N}^{+}(x) \cap \mathcal{N}^{+}(y)\right)=m_{\max } \times$ $\sigma(x) \wedge \sigma(y)$. Hence $\frac{\mu(x, y)}{(\sigma(x) \wedge \sigma(y))^{2}}=\frac{m_{\max }}{\sigma(x) \wedge \sigma(y)}>0.5$.

We have seen that if height of intersection between two out neighbourhoods of two vertices of a fuzzy digraph is greater than 0.5 , the edge between the two vertices in corresponding fuzzy competition graph is strong. This result is not true in corresponding fuzzy $k$-competition graph. A related result is presented below.

Theorem 3: Let $\vec{\xi}=(V, \sigma, \vec{\mu})$ be a fuzzy digraph. If $h\left(\mathcal{N}^{+}(x) \cap \mathcal{N}^{+}(y)\right)=1$ and $\left|\mathcal{N}^{+}(x) \cap \mathcal{N}^{+}(y)\right|>2 k$, then the edge $(x, y)$ is strong in $C_{k}(\vec{\xi})$.

Proof. Let $\vec{\xi}=(V, \sigma, \vec{\mu})$ be a fuzzy digraph and $C_{k}(\vec{\xi})=$ $(V, \sigma, \mu)$ be the corresponding fuzzy $k$-competition graph. Also let, $h\left(\mathcal{N}^{+}(x) \cap \mathcal{N}^{+}(y)\right)=1$ and $\left|\mathcal{N}^{+}(x) \cap \mathcal{N}^{+}(y)\right|>2 k$.

Now, $\mu(x, y)=\frac{k^{\prime}-k}{k^{\prime}} \sigma(x) \wedge \sigma(y) h\left(\mathcal{N}^{+}(x) \cap \mathcal{N}^{+}(y)\right)$, where $k^{\prime}=\left|\mathcal{N}^{+}(x) \cap \mathcal{N}^{+}(y)\right|$. So, $\mu(x, y)=\frac{k^{\prime}-k}{k^{\prime}} \sigma(x) \wedge \sigma(y)$. Hence $\frac{\mu(x, y)}{\sigma(x) \wedge \sigma(y)}=\frac{k^{\prime}-k}{k^{\prime}}>0.5$ as $k^{\prime}>2 k$. Hence the edge $(x, y)$ is strong.

\section{A. Fuzzy isolated vertex}

Isolated vertex in crisp graph is a vertex which has no incident edge i.e. a vertex of degree 0. In fuzzy graph, degree of a vertex is the sum of the membership values of incident edges. If the degree of a vertex in fuzzy graph is zero approximately, then the vertex can be assumed as isolated vertex. The formal definition of fuzzy isolated vertex is given below.

Definition 8: Let $\xi=(V, \sigma, \mu)$ be a fuzzy graph and $\epsilon$ be a pre-assigned positive real number. A vertex $x$ is said to be fuzzy isolated vertex if the degree of $x$ is less than $\epsilon$ i.e. $d(x)<\epsilon$.

An example of isolated vertex is given as follows.

Example 4: Let $\epsilon=0.05$. In Fig. 4, the degree of the vertex $b$ is $0.01+0.03=0.04$. As $d(b)<\epsilon, b$ is said to be fuzzy isolated vertex. By similar reason, $a$ and $c$ are fuzzy isolated vertices.

The fuzzy isolated vertices are not same like crisp isolated vertices. Fuzzy isolated vertices may have an edge of small strength. The definition of strong isolated vertices are given below. 


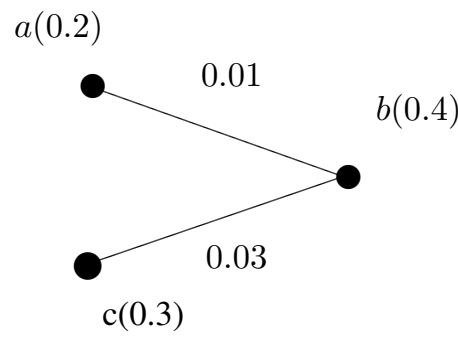

Fig. 4. An example of fuzzy isolated vertex.

Definition 9: Let $\xi=(V, \sigma, \mu)$ be a fuzzy graph. A vertex $x \in V$ is said to be strong isolated vertex in $\xi$ if $d(x)=0$.

In fuzzy graph, vertices which are not connected to any other vertices are called strong isolated vertices. So, strong isolated vertices are comparable with crisp isolated vertices.

\section{B. Fuzzy competition number}

Fuzzy competition number is associated with a fuzzy competition graph which is defined below.

Definition 10: Let $\vec{\xi}$ be an arbitrary fuzzy digraph and $C(\vec{\xi})$ be the corresponding fuzzy competition graph. The fuzzy competition number of a fuzzy graph $\psi$ is the minimum number of isolated fuzzy vertices such that $\psi$ along with these isolated fuzzy vertices (say $\xi$ ) is the fuzzy competition graph of $\vec{\xi}$ i.e. $C(\vec{\xi})=\xi$.

This concept is illustrated in the following example.

Example 5: Let $\psi=(V, \sigma, \mu)$ be a fuzzy graph (see Fig. 5(a)) with $V=\{(a, 0.4),(b, 0.6),(c, 0.1)\}$ and $E=$ $\{((a, b), 0.2),((b, c), 0.1),((c, a), 0.1)\}$. Now this fuzzy graph along with an isolated fuzzy vertex $d(0.4)$ and an edge $((c, d), 0.04)$ is drawn. Let $\xi=\psi \cup\{(d, 0.4),((c, d), 0.04)\}$. Now in Fig 5(b), a fuzzy multi-digraph $\vec{\xi}$ is shown. Clearly $C(\vec{\xi})=\xi$. Also, $d$ is said to be fuzzy isolated vertex if $\epsilon=0.05$. Thus the fuzzy competition number of $\psi$ is 1 .

An important theorem related to strong isolated vertices is given below.

Theorem 4: A path of length $m$ along with a strong isolated vertex may be a fuzzy competition graph of a fuzzy directed tree of $m+1$ vertices.

Proof. Let $P_{m}=(V, \sigma, \mu)$ be a fuzzy path where $V=$ $\left\{v_{1}, v_{2}, \ldots, v_{m}\right\}$. We take another vertex $u$ which is distinct from the vertices of $P_{m}$. So, the fuzzy path together with the strong isolated vertex has $m+1$ vertices. Now, we construct the directed fuzzy graph whose fuzzy competition graph is $P_{m} \cup\{u\}$. Keeping the concept of fuzzy competition graph of a fuzzy digraph, we construct the directed fuzzy edges $\overrightarrow{\left(v_{i}, u\right)}, i=1,2, \ldots, m$. Now this directed fuzzy graph is a directed fuzzy tree $T_{m+1}$ of $m+1$ vertices. Also, $C\left(T_{m+1}\right)=$ $P_{m} \cup\{u\}$. Hence the result.

The above theorem can be extended as follows. Some disjoint paths along with a strong isolated vertex may be a fuzzy competition graph of a fuzzy directed tree. Let the disjoint paths be $P_{m_{1}}, P_{m_{2}}, \ldots, P_{m_{n}}$, then the number of vertices of the corresponding directed fuzzy graph is

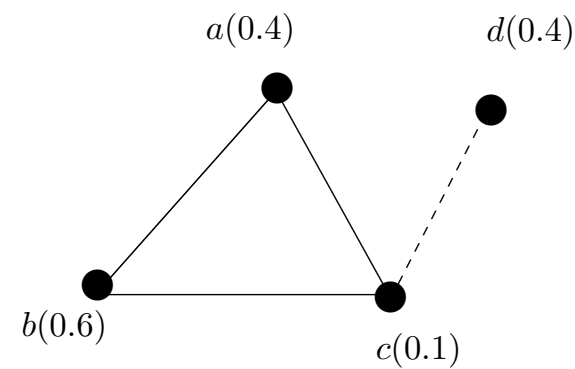

(a): $\psi \cup\{(d, 0.4),((c, d), 0.04)\}$

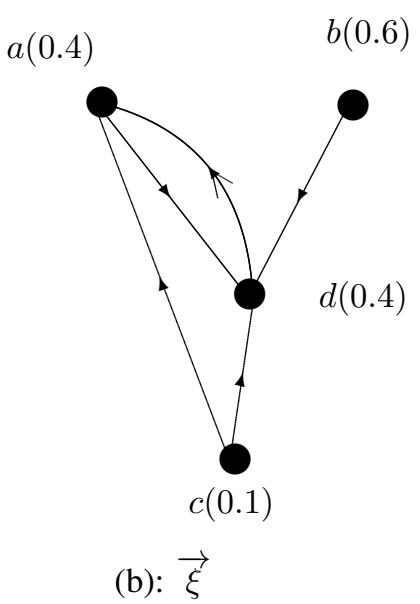

Fig. 5. Example of fuzzy competition number.

$m_{1}+m_{2}+\ldots+m_{n}+1$.

From Theorem 4, it is easy to observe that the fuzzy competition number of a path is 1 . Like crisp graph the fuzzy competition numbers can be found as follows.

The competition number of a fuzzy chordal graph which has no strong isolated vertex is 1 .

If a fuzzy graph is triangle free, then the fuzzy competition number is equal to two more than the difference between the number of edges and number of vertices.

The competition number of fuzzy complete graph is one.

\section{Fuzzy neighbourhood graphs}

The fuzzy open neighbourhood and fuzzy closed neighbourhood of a vertex in fuzzy graph are defined below.

Definition 11: Fuzzy open neighbourhood of a vertex $v$ of a fuzzy graph $\xi=(V, \sigma, \mu)$ is the fuzzy set $\mathcal{N}(v)=\left(X_{v}, m_{v}\right)$ where $X_{v}=\{u \mid \mu(v, u)>0\}$ and $m_{v}: X_{v} \rightarrow[0,1]$ defined by $m_{v}(u)=\mu(v, u)$. For each vertex $v \in V$, we define fuzzy singleton set, $A_{v}=\left(\{v\}, \sigma^{\prime}\right)$ such that $\sigma^{\prime}:\{v\} \rightarrow[0,1]$ defined by $\sigma^{\prime}(v)=\sigma(v)$. Fuzzy closed neighbourhood of a vertex $v$ is $\mathcal{N}[v]=\mathcal{N}(v) \cup A_{v}$.

In this section, fuzzy open neighbourhood graphs are defined and then fuzzy closed neighbourhood graphs. Based on these fuzzy graphs fuzzy $k$-neighbourhood graphs of open and closed types are defined. 
Definition 12: Let $\xi=(V, \sigma, \mu)$ be a fuzzy graph. Fuzzy open neighbourhood graph of $\xi$ is a fuzzy graph $\mathcal{N}(\xi)=$ $\left(V, \sigma, \mu^{\prime}\right)$ whose fuzzy vertex set is same as $\xi$ and has a fuzzy edge between two vertices $x$ and $y \in V$ in $\mathcal{N}(\xi)$ if and only if $\mathcal{N}(x) \cap \mathcal{N}(y)$ is non-empty fuzzy set in $\xi$ and $\mu^{\prime}: V \times V \rightarrow$ $[0,1]$ such that $\mu^{\prime}(x, y)=[\sigma(x) \wedge \sigma(y)] h(\mathcal{N}(x) \cap \mathcal{N}(y))$.

Definition 13: Let $\xi=(V, \sigma, \mu)$ be a fuzzy graph. Fuzzy closed neighbourhood graph of $\xi$ is a fuzzy graph $\mathcal{N}[\xi]=$ $\left(V, \sigma, \mu^{\prime}\right)$ whose fuzzy vertex set is same as $\xi$ and has a fuzzy edge between two vertices $x$ and $y \in V$ in $\mathcal{N}[\xi]$ if and only if $\mathcal{N}[x] \cap \mathcal{N}[y]$ is non-empty fuzzy set in $\xi$ and $\mu^{\prime}: V \times V \rightarrow$ $[0,1]$ such that $\mu^{\prime}(x, y)=[\sigma(x) \wedge \sigma(y)] h(\mathcal{N}[x] \cap \mathcal{N}[y])$.

Definition 14: Let $\xi=(V, \sigma, \mu)$ be a fuzzy graph. Fuzzy $(k)$-neighbourhood graph (read as open fuzzy $k$ neighbourhood graph) of $\xi$ is a fuzzy graph $\mathcal{N}_{k}(\xi)=$ $\left(V, \sigma, \mu^{\prime}\right)$ whose vertex set is same as $\xi$ and has an edge between two vertices $x$ and $y \in V$ in $\mathcal{N}_{k}(\xi)$ if and only if $|\mathcal{N}(x) \cap \mathcal{N}(y)|>k$ in $\xi$ and $\mu^{\prime}: V \times V \rightarrow[0,1]$ such that $\mu^{\prime}(x, y)=\frac{\left(k^{\prime}-k\right)}{k^{\prime}}[\sigma(x) \wedge \sigma(y)] h(\mathcal{N}(x) \cap \mathcal{N}(y))$ where $k^{\prime}=|\mathcal{N}(x) \cap \mathcal{N}(y)|$.

Definition 15: Let $\xi=(V, \sigma, \mu)$ be a fuzzy graph. Fuzzy $[k]$-neighbourhood graph (read as fuzzy closed $k$ neighbourhood graph) of $\xi$ is a fuzzy graph $\mathcal{N}_{k}[\xi]=\left(V, \sigma, \mu^{\prime}\right)$ whose fuzzy vertex set is same as $\xi$ and has a fuzzy edge between two vertices $x$ and $y \in V$ in $\mathcal{N}_{k}[\xi]$ if and only if $|\mathcal{N}[x] \cap \mathcal{N}[y]|>k$ in $\xi$ and $\mu^{\prime}: V \times V \rightarrow[0,1]$ such that $\mu^{\prime}(x, y)=\frac{\left(k^{\prime}-k\right)}{k^{\prime}}[\sigma(x) \wedge \sigma(y)] h(\mathcal{N}[x] \cap \mathcal{N}[y])$ where $k^{\prime}=|\mathcal{N}[x] \cap \mathcal{N}[y]|$.

Theorem 5: For every edge of a fuzzy graph $\xi$, there exists one edge in $\mathcal{N}[\xi]$.

Proof. Let $(x, y)$ be an edge of a fuzzy graph $\xi=(V, \sigma, \mu)$. Let the corresponding closed neighbourhood graph be $\mathcal{N}[\xi]=$ $(V, \sigma, \nu)$. Then $x, y \in \mathcal{N}[x]$ and $x, y \in \mathcal{N}[y]$. So $x, y \in \mathcal{N}[x] \cap$ $\mathcal{N}[y]$. Hence $h(\mathcal{N}[x] \cap \mathcal{N}[y]) \neq 0$. Now, $\nu(x, y)=\sigma(x) \wedge$ $\sigma(y) h(\mathcal{N}[x] \cap \mathcal{N}[y]) \neq 0$. So for every edge $(x, y)$ in $\xi$, there exists an edge $(x, y)$ in $\mathcal{N}[\xi]$.

Definition 16: Let $\vec{\xi}=(V, \sigma, \vec{\mu})$ be a fuzzy digraph. The underlying fuzzy graph of $\vec{\xi}$ is denoted by $\mathcal{U}(\xi)$ and is defined as $\mathcal{U}(\xi)=(V, \sigma, \mu)$ where $\mu(u, v)=\min \{\vec{\mu}(u, v), \vec{\mu}(v, u)\}$ for all $u, v \in V$.

A relation between fuzzy $(k)$-neighbourhood graph and fuzzy $k$-competition graph is established below.

Theorem 6: If the symmetric fuzzy digraph $\vec{\xi}$ is loop less, $\mathcal{C}_{k}(\vec{\xi})=\mathcal{N}_{k}(\mathcal{U}(\xi))$ where $\mathcal{U}(\xi)$ is the fuzzy graph underlying $\vec{\xi}$.

Proof. Let a directed fuzzy graph be $\vec{\xi}=(V, \sigma, \vec{\mu})$ and the corresponding underlying fuzzy graph be $\mathcal{U}(\xi)=(V, \sigma, \mu)$. Also let $\mathcal{C}_{k}(\vec{\xi})=(V, \sigma, \nu)$ and $\mathcal{N}_{k}(\mathcal{U}(\xi))=\left(V, \sigma, \nu^{\prime}\right)$. The fuzzy vertex set of $\vec{\xi}$ is equal to $\mathcal{C}_{k}(\vec{\xi})$. Also an underlying fuzzy graph has the same fuzzy vertex set as the directed fuzzy graph. Hence $\mathcal{N}_{k}(\mathcal{U}(\xi))$ has the same fuzzy vertex set as $\vec{\xi}$. Now we need to show that $\nu(x, y)=\nu^{\prime}(x, y)$ for all $x, y \in V$. If $\nu(x, y)=0$ in $\mathcal{C}_{k}(\vec{\xi})$ then $\left|\mathcal{N}^{+}(x) \cap \mathcal{N}^{+}(y)\right| \leq k$. As $\vec{\xi}$ is symmetric fuzzy set, $|\mathcal{N}[x] \cap \mathcal{N}[y]| \leq k$ in $\mathcal{U}(\xi)$. So $\nu^{\prime}(x, y)=0$.
If $\left|\mathcal{N}^{+}(x) \cap \mathcal{N}^{+}(y)\right|>k$ then $\nu(x, y)>0$ in $\mathcal{C}_{k}(\vec{\xi})$. So $\nu(x, y)=\frac{\left(k^{\prime}-k\right)}{k^{\prime}}[\sigma(x) \wedge \sigma(y)] h\left(\mathcal{N}^{+}(x) \cap \mathcal{N}^{+}(y)\right)$ where $k^{\prime}=\left|\mathcal{N}^{+}(x) \cap \mathcal{N}^{+}(y)\right|$. As $\vec{\xi}$ is symmetric fuzzy digraph, $|\mathcal{N}[x] \cap \mathcal{N}[y]|>k$ in $\mathcal{U}(\xi)$. So $\nu^{\prime}=\frac{\left(k^{\prime \prime}-k\right)}{k^{\prime \prime}}[\sigma(x) \wedge$ $\sigma(y)] h(\mathcal{N}[x] \cap \mathcal{N}[y])$ where $k^{\prime \prime}=|\mathcal{N}[x] \cap \mathcal{N}[y]|$. It is clear that $h\left(\mathcal{N}^{+}(x) \cap \mathcal{N}^{+}(y)\right)$ in $\vec{\xi}$ is equal to $h(\mathcal{N}[x] \cap \mathcal{N}[y])$ in $\mathcal{U}(\xi)$ as $\vec{\xi}$ is symmetric. $k^{\prime}=k^{\prime \prime}$ for similar reason. Hence $\nu(x, y)=\nu^{\prime}(x, y)$ for all $x, y \in V$.

Similarly, a relation between fuzzy $[k]$-neighbourhood graph and fuzzy $k$-competition graph is established below.

Theorem 7: If the symmetric fuzzy digraph $\vec{\xi}$ has loop at every vertex, then $\mathcal{C}_{k}(\vec{\xi})=\mathcal{N}_{k}[\mathcal{U}(\xi)]$ where $\mathcal{U}(\xi)$ is the loop less fuzzy graph underlying $\vec{\xi}$.

Proof. Let a directed fuzzy graph be $\vec{\xi}=(V, \sigma, \vec{\mu})$ and the corresponding underlying loop less graph be $\mathcal{U}(\xi)=(V, \sigma, \mu)$. Also let $\mathcal{C}_{k}(\vec{\xi})=(V, \sigma, \nu)$ and $\mathcal{N}_{k}[\mathcal{U}(\xi)]=\left(V, \sigma, \nu^{\prime}\right)$. The fuzzy vertex set of $\vec{\xi}$ is equal to $\mathcal{C}_{k}(\vec{\xi})$. Also an underlying fuzzy graph has the same fuzzy vertex set as the directed fuzzy graph. Hence $\mathcal{N}_{k}[\mathcal{U}(\xi)]$ has the same fuzzy vertex set as $\vec{\xi}$. Now we need to show that $\nu(x, y)=\nu^{\prime}(x, y)$ for all $x, y \in V$. As the directed fuzzy graph $\vec{\xi}$ has a loop at every vertex, out-neighbourhood of each vertex contains the vertex itself. Hence if $\nu(x, y)=0$ in $\mathcal{C}_{k}(\vec{\xi})$ then $\left|\mathcal{N}^{+}(x) \cap \mathcal{N}^{+}(y)\right| \leq k$. As $\vec{\xi}$ is symmetric fuzzy set, $|\mathcal{N}(x) \cap \mathcal{N}(y)| \leq k$ in $\mathcal{U}(\xi)$. So $\nu^{\prime}(x, y)=0$.

If $\left|\mathcal{N}^{+}(x) \cap \mathcal{N}^{+}(y)\right|>k$ then $\nu(x, y)>0$ in $\mathcal{C}_{k}(\vec{\xi})$. So $\nu(x, y)=\frac{\left(k^{\prime}-k\right)}{k^{\prime}}[\sigma(x) \wedge \sigma(y)] h\left(\mathcal{N}^{+}(x) \cap \mathcal{N}^{+}(y)\right)$ where $k^{\prime}=$ $\left|\mathcal{N}^{+}(x) \cap \mathcal{N}^{+}(y)\right|$. As $\vec{\xi}$ is symmetric fuzzy digraph, $\mid \mathcal{N}(x) \cap$ $\mathcal{N}(y) \mid>k$ in $\mathcal{U}(\xi)$. So $\nu^{\prime}=\frac{\left(k^{\prime \prime}-k\right)}{k^{\prime \prime}}[\sigma(x) \wedge \sigma(y)] h(\mathcal{N}(x) \cap$ $\mathcal{N}(v))$ where $k^{\prime \prime}=|\mathcal{N}(x) \cap \mathcal{N}(y)|$. It is clear that $h\left(\mathcal{N}^{+}(x) \cap\right.$ $\left.\mathcal{N}^{+}(y)\right)$ in $\vec{\xi}$ is equal to $h(\mathcal{N}(x) \cap \mathcal{N}(v))$ in $\mathcal{U}(\xi)$ and $k^{\prime}=k^{\prime \prime}$ as $\vec{\xi}$ is symmetric. Hence $\nu(x, y)=\nu^{\prime}(x, y)$ for all $x, y \in$ $V$.

\section{ISOMORPHISM IN FUZZY COMPETITION GRAPH}

Isomorphism on fuzzy graphs are well known in literature. Here the isomorphism in fuzzy digraphs are introduced. A homomorphism of fuzzy digraphs $\vec{\xi}$ and $\overrightarrow{\xi^{\prime}}$ is a map $h$ : $S \rightarrow S^{\prime}$ which satisfies $\sigma(x) \leq \sigma^{\prime}(h(x))$ for all $x \in S$ and $\mu \overrightarrow{(x, y)} \leq \mu^{\prime} \overrightarrow{(h(x), h(y))}$ for all $x, y \in S$ where $S$ is the set of vertices of $\vec{\xi}$ and $S^{\prime}$ is that of $\overrightarrow{\xi^{\prime}}$.

A weak isomorphism between fuzzy digraphs is a bijective homomorphism $h: S \rightarrow S^{\prime}$ which satisfies $\sigma(x)=\sigma^{\prime}(h(x))$ for all $x \in S$.

A co- weak isomorphism between fuzzy digraphs is a bijective homomorphism $h: S \rightarrow S^{\prime}$ which satisfies $\mu \overrightarrow{(x, y)}=$ $\mu^{\prime} \overrightarrow{(h(x), h(y))}$ for all $x, y \in S$.

An isomorphism between fuzzy graphs of fuzzy digraphs is a bijective homomorphism $h: S \rightarrow S^{\prime}$ which satisfies $\sigma(x)=$ $\sigma^{\prime}(h(x))$ for all $x \in S$ and $\mu \overrightarrow{(x, y)}=\mu^{\prime} \overrightarrow{(h(x), h(y))}$ for all $x, y \in S$.

Isomorphism between fuzzy graphs is an equivalence relation. But, if there is an isomorphism between two fuzzy 


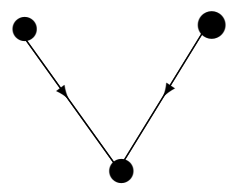

(a): $\overrightarrow{\xi_{1}}$

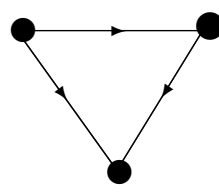

(b): $\overrightarrow{\xi_{2}}$

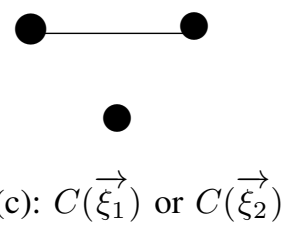

Fig. 6. Fuzzy competition graphs are isomorphic but corresponding digraphs are not isomorphic.

graph and one is fuzzy competition graph, then the other will be fuzzy competition graph. But, the corresponding digraphs may not be isomorphic. This result can be shown as follows.

Remark 1: Let $\overrightarrow{\xi_{1}}$ and $\overrightarrow{\xi_{2}}$ be two digraphs such that $C\left(\overrightarrow{\xi_{1}}\right)$ and $C\left(\overrightarrow{\xi_{2}}\right)$ are isomorphic. But $\overrightarrow{\xi_{1}}$ and $\overrightarrow{\xi_{2}}$ may not be isomorphic.

This remark can be illustrated from the following example. In Fig. 6(a) and Fig. 6(b), two fuzzy digraphs are shown. These fuzzy digraphs are not isomorphic as degree of vertices of these fuzzy digraphs are not same. But, the corresponding fuzzy competition graphs are isomorphic.

If two fuzzy digraphs are isomorphic, their corresponding fuzzy competition graphs must be isomorphic. This can established in the following theorem.

Theorem 8: If two fuzzy digraphs $\overrightarrow{\xi_{1}}$ and $\overrightarrow{\xi_{2}}$ are isomorphic, then $C\left(\overrightarrow{\xi_{1}}\right)$ and $C\left(\overrightarrow{\xi_{2}}\right)$ are isomorphic.

Proof. Here two fuzzy digraphs $\overrightarrow{\xi_{1}}$ and $\overrightarrow{\xi_{2}}$ are isomorphic. Then there exists one to one correspondence between vertices and edges. Also the membership values of the vertices and edges are preserved. So the adjacency of edges are preserved. Then it is easy to observe that $C\left(\overrightarrow{\xi_{1}}\right)$ and $C\left(\overrightarrow{\xi_{2}}\right)$ are isomorphic.

Similarly, we can prove that if two fuzzy digraphs $\overrightarrow{\xi_{1}}$ and $\overrightarrow{\xi_{2}}$ are isomorphic, then $C_{k}\left(\overrightarrow{\xi_{1}}\right)$ and $C_{k}\left(\overrightarrow{\xi_{2}}\right)$ are isomorphic.

\section{CONCLusions}

This study introduces fuzzy competition graphs and fuzzy $k$ competition graph. In these fuzzy graphs, if there is a common out neighbourhood of two vertices, then there will be an edge between the vertices. Thus the fuzzy competition graph can be stated as 1-step fuzzy competition graph. In future, $m$-step fuzzy competition graphs can be investigated as an extension of this study. Besides, fuzzy isolated vertices and fuzzy competition numbers are introduced. The competition numbers of paths and several graphs are investigated. But, fuzzy competition number of any fuzzy graphs is not calculated here. This can be investigated in future. Isomorphism in fuzzy graphs is new in the research field. Isomorphism relation between two fuzzy competition graphs are established. Also, some fundamental results related to fuzzy competition graphs are presented. These results will be helpful in ecosystem, competitive markets, etc.

\section{REFERENCES}

[1] B. D. Acharya and M. N. Vartak, Open neighbourhood graphs, Research Report 07, IIT Bombay, 1973.

[2] M. Akram and W. A. Dudek, Interval-valued fuzzy graphs, Computers and Mathematics with Applications, 61(2), 289-299, 2011.

[3] M. Akram, W. A. Dudek, Intuitionistic fuzzy hypergraphs with applications, Information Sciences, 218, 182-193, 2013.

[4] M. Akram, Bipolar fuzzy graphs with applications, Knowledge Based System, 39, 1-8, 2013.

[5] K. R. Bhutani and A. Battou, On M-strong fuzzy graphs, Information Sciences, 155(12), 103-109, 2003.

[6] K. R. Bhutani and A. Rosenfeld, Strong arcs in fuzzy graphs, Information Sciences, 152, 319-322, 2003.

[7] K. R. Bhutani, J. Moderson and A. Rosenfeld, On degrees of end nodes and cut nodes in fuzzy graphs, Iranian Journal of Fuzzy Systems, 1(1), 57-64, 2004.

[8] R. C. Brigham and R. D. Dutton, On neighbourhood graphs, Journal of Combinatories, Information, and System Sciences, 12, 75-85, 1987.

[9] R.C. Brigham, F. R. McMorris and R. P. Vitray, Tolerance competition graphs, Linear Algebra and its Application, 217, 41-52, 1995.

[10] C. Cable, K. F. Jones, J. R. Lundgren and S. Seager, Niche graphs, Discrete Apllied Mathematics, 23(3), 231-241, 1989.

[11] H. H. Cho, S. R. Kim and Y. Nam, The $m$-step competition graph of a diagraph, Discrete Applied Mathematics, 105(1-3), 115-127, 2000.

[12] J. E. Cohen, Interval graphs and food webs: a finding and a problem, Document 17696-PR, RAND Corporation, Santa Monica, CA (1968).

[13] C. Eslahchi and B. N. Onaghe, Vertex Strength of Fuzzy Graphs, International Journal of Mathematics and Mathematical Sciences, Volume 2006, Article ID 43614, Pages 1-9, DOI 10.1155/IJMMS/2006/43614.

[14] P. Ghosh, K. Kundu and D. Sarkar, Fuzzy graph representation of a fuzzy concept lattice, Fuzzy Sets and Systems, 161(12), 1669-1675, 2010

[15] M. C. Gulumbic and A. Trenk, Tolerance Graphs, Cambridge University Press, (2004).

[16] G. Isaak, S. R. Kim, T. A. McKee, F.R. McMorris and F. S. Roberts, 2-competition graphs, SIAM J. Disc. Math., 5(4), 524-538, 1992.

[17] J. B. Jenson and G. Z. Gutin, Digraphs: Theory, Algorithms and Applications, Springer-verlag, 2009.

[18] S. R. Kim, Graphs with one hole and competition number one, J. Korean Math. Soc, 42(6), 1251-1264, 2005.

[19] S. R. Kim, T. A. McKee, F.R. McMorris and F. S. Roberts, p-competition graphs, Linear Algebra and its Application, 217, 167-178, 1995.

[20] L. T. Koczy, Fuzzy graphs in the evaluation and optimization of networks, Fuzzy Sets and Systems, 46, 307-319, 1992.

[21] J. R. Lundgren and J. S. Maybee, Food webs with interval competition graph, In Graphs and Applcations: Proceedings of the first colorado symposium on graph theory, Wiley, Newyork, 1984.

[22] S. Mathew and M.S. Sunitha, Types of arcs in a fuzzy graph, Information Sciences, 179, 1760-1768, 2009.

[23] S. Mathew and M.S. Sunitha, Node connectivity and arc connectivity of a fuzzy graph, Information Sciences, 180(4), 519-531, 2010.

[24] J. N. Mordeson and P. S. Nair, Fuzzy graphs and hypergraphs, Physica Verlag, 2000.

[25] J. N. Mordeson and P. S. Nair, Successor and source of (fuzzy) finite state machines and (fuzzy) directed graphs, Information Sciences, 95(12), 113-124, 1996.

[26] S. Muoz, M. T. Ortuo, J. Ramirez and J. Yez, Coloring fuzzy graphs, Omega, 33(3), 211-221, 2005.

[27] A. Nagoorgani and K. Radha, On regular fuzzy graphs, Journal of Physical Sciences, 12, 33-40, 2008.

[28] A. Nagoorgani and J. Malarvizhi, Isomorphism properties of strong fuzzy graphs, International Journal of Algorithms, Computing and Mathematics, 2(1), 39-47, 2009.

[29] A. Nagoorgani and P. Vadivel, Relations between the parameters of independent domination and irredundance in fuzzy graph, International Journal of Algorithms, Computing and Mathematics, 2(1), 15-19, 2009. 
[30] A. Nagoorgani and P. Vijayalaakshmi, Insentive arc in domination of fuzzy graph, Int. J. Contemp. Math. Sciences, 6(26), 1303-1309, 2011.

[31] A. Nagoorgani and R. J. Hussain, Fuzzy effective distance $k$-dominating sets and their applications, International Journal of Algorithms, Computing and Mathematics, 2(3), 25-36, 2009.

[32] P. S. Nair and S. C. Cheng, Cliques and fuzzy cliques in fuzzy graphs, IFSA World Congress and 20th NAFIPS International Conference, 4 2277 - 2280, 2001.

[33] P. S. Nair, Perfect and precisely perfect fuzzy graphs, Fuzzy Information Processing Society, 19-22, 2008.

[34] C. Natarajan and S. K. Ayyasawamy, On strong (weak) domination in fuzzy graphs, World Academy of Science, Engineering and Technology, 67, 247-249, 2010.

[35] A. Raychaudhuri and F. S. Roberts, Generalized competition graphs and their applications, IX symposium on operations research, Part I, Sections 14 (Osnabruck, 1984) Athenaum/Hain/Hanstein, Konigstein, Methods Oper. Res. 49,295-311, 1985.

[36] A. Rosenfeld, Fuzzy graphs, in: L.A. Zadeh, K.S. Fu, M. Shimura (Eds.), Fuzzy Sets and Their Applications, Academic Press, New York, 77-95, 1975.

[37] S. Samanta and M. Pal, Fuzzy tolerance graphs, International Journal of Latest Trends in Mathematics, 1(2), 57-67, 2011.

[38] S. Samanta and M. Pal, Fuzzy threshold graphs, CIIT International Journal of Fuzzy Systems, 3(12), 360-364, 2011.

[39] S. Samanta and M. Pal, Irregular bipolar fuzzy graphs, Inernational Journal of Applications of Fuzzy Sets, 2, 91-102, 2012.

[40] S. samanta and M. Pal, Bipolar fuzzy hypergraphs, International Journal of Fuzzy Logic Systems, 2(1), 17 - 28, 2012.

[41] S. Samanta, M. Pal and A. Pal, Some more results on bipolar fuzzy sets and bipolar fuzzy intersection graphs, To appear in The Journal of Fuzzy Mathematics.

[42] S. Samanta and M. Pal, A new approach to social networks based on fuzzy graphs, To appear in Journal of Mass Communication and Journalism.

[43] Y. Sano, The competition-common enemy graphs of digraphs satisfying Conditions $C(p)$ and $C^{\prime}(p)$, arXiv:1006.2631v2 [math.CO], 2010.

[44] Y. Sano, Characterizations of competition multigraphs, Discrete Applied Mathematics, 157(13), 2978-2982, 2009.

[45] D. D. Scott, The competition-common enemy graph of a digraph, Discrete Appl. Math., 17, 269-280, 1987.

[46] M. Sonnatag and H. M. Teichert, Competition hypergraphs, Discrete Appl. Math., 143, 324-329, 2004.

[47] A. Somasundaram and S. Somasundaram, Domination in fuzzy graphs1, Pattern Recognition Letters, 19(9), 787-791, 1998. 